\title{
Strong Convergence of the Hybrid Halpern Type Proximal Point Algorithm
}

\author{
Liu Liu ${ }^{1}$, Qing-bang Zhang ${ }^{2, *}$ \\ ${ }^{1}$ Department of Mathematics, Sichuan University of Arts and Science, Dazhou, P.R.China \\ ${ }^{2}$ College of Economic Mathematics, Southwestern University of Finance and Economics, Chengdu, P.R.China
}

\section{Email address:}

dcliuliu@163.com (Liu Liu), zhangqingbang@126.com (Qing-bang Zhang), zhangqb@ swufe.edu.cn (Qing-bang Zhang)

${ }^{*}$ Corresponding author

\section{To cite this article:}

Liu Liu, Qing-bang Zhang. Strong Convergence of the Hybrid Halpern Type Proximal Point Algorithm. Applied and Computational Mathematics. Vol. 9, No. 6, 2020, pp. 187-194. doi: 10.11648/j.acm.20200906.13

Received: May 15, 2020; Accepted: June 12, 2020; Published: November 16, 2020

\begin{abstract}
Based on the proximal point algorithm, which is a widely used tool for solving a variety of convex optimization problems, there are many algorithms for finding zeros of maximally monotone operators. The algorithm works by applying successively so-called "resolvent" mappings with errors associated to the original object, and is weakly convergent in Hilbert space. In order to acquiring the strong convergence of the algorithm, in this paper, we construct a hybrid Halpern type proximal point algorithm with errors for approximating the zero of a maximal monotone operator, which is a combination of modified proximal point algorithm raised by Yao and Noor and Halpern inexact proximal point algorithm raised by Zhang, respectively. Then, we prove the strong convergence of our algorithm with weaker assumptions in Hilbert space. Finally, we present a numerical example to show the convergence and the convergence speed, which is not affected but accelerated by the projection in the algorithm. Our work improved and generalized some known results.
\end{abstract}

Keywords: Hybrid Halpern Type Proximal Point Algorithm, Maximal Monotone Operator, Strong Convergence, Hilbert Space

\section{Introduction}

Let $\mathcal{H}$ be a real Hilbert space endowed with a norm $\|\cdot\|$ and an inner product $\langle\cdot, \cdot\rangle$, respectively, $2^{\mathcal{H}}$ denote the family of all the nonempty subsets of $\mathcal{H}$. Let $M: \mathcal{H} \rightarrow 2^{\mathcal{H}}$ be a set-valued mapping, $S$ denote the set of all zeros of $M$, i.e., $S=\{x \in \mathcal{H}: 0 \in M(x)\}$. Throughout this paper, let $M$ be maximal monotone and $S \neq \emptyset$. It is known that $S$ is closed and convex. For any $k>0$, we use $J_{k}$ to denote the resolvent of $M$, i.e., $J_{k}=\left(I+\lambda_{k} M\right)^{-1}$, where $I$ denotes the identity mapping on $\mathcal{H}$.

We consider the problem: Find $x \in \mathcal{H}$ such that

$$
0 \in M(x) .
$$

It's well known that many problems in nonlinear analysis and optimization can be formulated as the equation (1).

To solve this problem, many researchers have been studied some interesting methods. Rockafellar [1] introduced a fundamental inexact proximal point algorithm and proved the sequence $\left\{x^{k}\right\}$, generated from an initial point $x^{0}$ by

$$
x^{k+1}=J_{k}\left(x^{k}+e^{k}\right),
$$

converges weakly to a solution of (1) in $R^{n}$ if $\lambda_{k} \geq c>0$, where $\left\{e^{k}\right\}$ is an error sequence. Furthermore, he posed an open question of whether the sequence generated by (2) converges strongly. In 1991, Güler [2] gave an example and showed that Rockafellar' algorithm (2) only had weak convergence even in an infinity-dimensional Hilbert space.

In 1992, Eckstein and Bertsekas [3] proved that the sequence $\left\{x^{k}\right\}$, generated from an initial point $x^{0}$ by

$$
x^{k+1}=\left(1-\rho_{k}\right) x^{k}+\rho_{k} w_{k}, \forall k \geq 0,
$$

where $\left\|w_{k}-J_{k}\left(x^{k}\right)\right\| \leq \varepsilon_{k}$ for sequences $\left\{\varepsilon_{k}\right\}_{k=0}^{\infty},\left\{\rho_{k}\right\}_{k=0}^{\infty}$, 
$\left\{\lambda_{k}\right\}_{k=0}^{\infty}$ satisfying

$$
\sum_{k=0}^{\infty} \varepsilon_{k}<\infty, \quad \inf _{k \geq 0} \rho_{k}>0, \quad \sup _{k \geq 0} \rho_{k}<2 \text { and } \inf _{k \geq 0} \lambda_{k}>0
$$

converges weakly to a solution to (1) in Hilbert spaces, which is called the generalized proximal point algorithm.

Recently, by using a modified orthogonal projection onto a moving halt-spaces, which inspired by the projection onto a given closed convex set $\Omega$ (or $C$ ) in [4] and [5], Zhang [6] constructed a modified proximal point algorithm with errors for approximating the solution of Problem (1) as follows:

$$
\begin{cases}x^{0} & \in \mathcal{H}, \\ y^{k} & =J_{k}\left(x^{k}+e^{k}\right), \\ x^{k+1} & =\left(1-\beta_{k}\right) x^{k}+\beta_{k} P_{K}\left(x^{k}-\rho_{k}\left(x^{k}-y^{k}\right)\right),\end{cases}
$$

where $K=\left\{z \in \mathcal{H}:\left\langle x^{k}-y^{k}+e^{k}, z-y^{k}\right\rangle \leq 0\right\}$, and showed its weak convergence in Hilbert space. The weak convergence has being extended to Banach space by Liu [7] in 2015, but the strong convergence of the algorithm is not given.

To obtain the strong convergence of the iterative algorithm for solving Problem (1), there are some results in [8]-[15]. It is worthy to mention some of the works. A simple modification of Halpern iterative scheme is provided by Kim and $\mathrm{Xu}$ [10] and $\mathrm{Xu}[14]$ in either a uniformly smooth Banach space or a reflexive Banach space $E$ as follows:

$$
\begin{cases}x^{0} & =x \in E \\ x^{k+1} & =\alpha_{k} u+\left(1-\alpha_{k}\right) J_{k}\left(x^{k}\right)\end{cases}
$$

where $u \in \overline{D(A)} \subset E$ is an arbitrary element.

Tian and Song [12] improved the results offered by Xu [16], and proved the iterative sequence $\left\{x^{k}\right\}$ generated by

$$
x^{k+1}=J_{k}\left(\alpha_{k} u+\left(1-\alpha_{k}\right) x^{k}+e^{k}\right), k \geq 0,
$$

or

$$
x^{k+1}=\alpha_{k} u+\left(1-\alpha_{k}\right) J_{k}\left(x^{k}\right)+e^{k}, k \geq 0,
$$

converges strongly to $P_{S}(u)$, where $u$ is given, $\left\{\alpha_{k}\right\} \subset(0,1)$ satisfying

$\lim _{k \rightarrow \infty} \alpha_{k}=0, \sum_{k=0}^{\infty} \alpha_{k}=\infty, \liminf _{k \rightarrow \infty} \lambda_{k}>0, \sum_{k=0}^{\infty}\left\|e_{k}\right\|<\infty$.

Yao and Noor [15] suggested the following generalized version of the contraction proximal point algorithm: for given $u \in \mathcal{H}$, the sequence $\left\{x^{k}\right\}$ generated by

$$
x^{k+1}=\alpha_{k} u+\gamma_{k} x^{k}+\beta_{k} J_{k}\left(x^{k}\right)+e^{k}, k \geq 0,
$$

where $\alpha_{k}, \beta_{k}, \gamma_{k} \subset(0,1)$ with $\alpha_{k}+\beta_{k}+\gamma_{k}=1(k \geq 0)$, and proved its strong convergence with the assumptions:

$$
\lim _{k \rightarrow \infty} \alpha_{k}=0, \sum_{k=0}^{\infty} \alpha_{k}=\infty, \quad 0<\liminf _{k \rightarrow \infty} \gamma_{k} \leq \limsup _{k \rightarrow \infty} \gamma_{k}<1,
$$

$$
\lambda_{k} \geq c(\text { a positive constant }), \lambda_{k+1}-\lambda_{k} \rightarrow 0, \sum_{k=0}^{\infty}\left\|e_{k}\right\|<\infty \text {. }
$$

Zhang and Song [17] proved the strong convergence of Algorithm (8) in a uniformly convex Banach space with a uniformly Gâteaux differentiable norm for approximating the zero of a m-accretive operator.

Based on the work of Yao and Noor [15] and the work of Zhang [6], we will construct a hybrid Halpern type proximal point algorithm with errors, which is a combination of modified proximal point algorithm and Halpern inexact proximal point algorithm, to find a solution of Problem (1) involving a maximal monotone operator $M$, and prove its strong convergence. To show the effectiveness of the algorithm, an example will be given. Our work would improve and generalize the corresponding results of Yao and Noor [15], Zhang [6], Khatibzadeh and Ranjbar [9] and some others.

\section{Preliminaries}

Let $\mathcal{H}$ be a real Hilbert space endowed with a norm $\|\cdot\|$ and an inner product $\langle\cdot, \cdot\rangle$. The strong and weak convergence are denoted by $\rightarrow$ and $\rightarrow$.

An operator $M: \mathcal{H} \rightarrow 2^{\mathcal{H}}$ is monotone if $\langle x-y, u-v\rangle \geq 0$, for all $(x, u),(y, v) \in G(M) \doteq\{(z, w) \in \mathcal{H} \times \mathcal{H}: w \in$ $M(z)\}$. A monotone operator $M: \mathcal{H} \rightarrow 2^{\mathcal{H}}$ is maximal if there exists no monotone operator $F: \mathcal{H} \rightarrow 2^{\mathcal{H}}$ such that $G(M) \subset G(F)$. The following assertion can be obtained directly from the definition above: for a maximal monotone operator $M: \mathcal{H} \rightarrow 2^{\mathcal{H}}$ and $(x, u) \in G(M)$,

$$
(x, u) \in G(M) \Longleftrightarrow\langle x-z, u-w\rangle \geq 0(\forall(z, w) \in G(M)) .
$$

In other words, an operator $M: \mathcal{H} \rightarrow 2^{\mathcal{H}}$ is maximal monotone if and only if $M$ is monotone and $(I+\lambda M)(\mathcal{H})=$ $\mathcal{H}$ for all $\lambda>0$.

The following property and lemma will be needed for the proof of main results.

For any $k>0$, the resolvent $J_{k}=\left(I+\lambda_{k} M\right)^{-1}$ has the following property that(see [18], [19]):

(i) the resolvent $J_{k}$ is firmly non-expansive, i.e.,

$$
\left\|J_{k}(x)-J_{k}(y)\right\| \leq\|x-y\|-\left\|\left(x-J_{k}(x)\right)-\left(y-J_{k}(y)\right)\right\| .
$$

(ii) $S=F i x\left(J_{k}\right):=\left\{x \in X \mid J_{k}(x)=x\right\}$ for any $k>0$;

(iii) If $0<k_{1} \leq k_{2}$, then $\left\|J_{k_{1}}(x)-x\right\| \leq\left\|J_{k_{2}}(x)-x\right\|$, $\forall x \in \mathcal{H}$;

(iv) for $\lambda, \mu>0$, the identity $J_{\lambda}(x)=J_{\mu}\left(\frac{\mu}{\lambda} x+(1-\right.$ $\left.\left.\frac{\mu}{\lambda}\right) J_{\lambda}(x)\right), \forall x \in \mathcal{H}$.

Let $P_{X}(z)$ denote the projection of $z$ onto a closed convex subset $X \subset \mathcal{H}$, that is, a unique element $P_{X}(z) \in X$ satisfies the condition

$$
\left\|z-P_{X}(z)\right\|=\operatorname{dist}(z, X):=\inf _{x \in X}\|z-x\| .
$$


Then we have the following well-known properties of the projection operator. For any $x, y \in \mathcal{H}$ and $z \in X$,

(P1) $u=P_{X}(x) \Longleftrightarrow\langle u-x, z-u\rangle \geq 0$.

(P2) $\left\|P_{X}(x)-P_{X}(y)\right\| \leq\|x-y\|$.

(P3) $\left\|P_{X}(x)-z\right\|^{2} \leq\|x-z\|^{2}-\left\|P_{X}(x)-x\right\|^{2}$.

Lemma 2.1. ([20]) Let $\left\{a_{n}\right\}$ be a sequence of nonnegative real numbers satisfying the property

$$
a_{n+1} \leq\left(1-\tau_{n}\right) a_{n}+\tau_{n} t_{n}+\delta_{n}, \quad n \geq 0,
$$

where $\left\{\tau_{n}\right\} \subset(0,1)$ and $\left\{t_{n}\right\}$ are such that

$$
\sum_{n=0}^{\infty} \tau_{n}=\infty
$$

either

$$
\limsup _{n \rightarrow \infty} t_{n} \leq 0 \text { or } \sum_{n=0}^{\infty}\left|\tau_{n} t_{n}\right|<\infty, \quad \sum_{n=0}^{\infty} \delta_{n}<\infty .
$$

Then $\lim _{n \rightarrow \infty} a_{n}=0$.

Lemma 2.2. ([21]) Let $\left\{s_{n}\right\}$ be a real sequence that does not decrease at infinity, i.e., there exists a subsequence $\left\{s_{n_{j}}\right\}$ so that $s_{n_{j}} \leq s_{n_{j}+1}$ for all $j \geq 0$.

For every $n>n_{0}$ define an integer sequence $\left\{\tau_{n}\right\}$ as

$$
\tau_{n}=\max \left\{n_{0} \leq j \leq n: s_{j}<s_{j+1}\right\} .
$$

Then $\tau_{n} \rightarrow \infty$ as $n \rightarrow \infty$ and for all $n>n_{0}$, $\max \left(s_{\tau_{n}}, s_{n}\right) \leq s_{\tau_{n}+1}$.

\section{Algorithm and Convergence}

In this section, we will propose a Hybrid Halpern type proximal point algorithm, which is a combination of modified proximal point algorithm and Halpern inexact proximal point algorithm, and prove the strong convergence of the iterative sequence $\left\{x^{k}\right\}$ generated by the given algorithm.

Algorithm 3.1. For a given point $u \in \mathcal{H}$.

Step 1. Select initial $x^{0} \in \mathcal{H}$ and set $k=0$.

Step 2. Find $y^{k} \in \mathcal{H}$ such that

$$
y^{k}=J_{k}\left(x^{k}+e^{k}\right),
$$

where a positive sequence $\left\{\lambda_{k}\right\}$ satisfies $\liminf _{k \geq 0} \lambda_{k}=\alpha>0$ and $\left\{e^{k}\right\}$ is an error sequence.

Step 3. Set $C_{k}=\left\{z \in \mathcal{H}:\left\langle x^{k}-y^{k}+e^{k}, z-y^{k}\right\rangle \leq 0\right\}$, choose a real sequence $\rho_{k} \in[0, \infty)$ and compute

$$
t^{k}=P_{k}\left(x^{k}-\rho_{k}\left(x^{k}-y^{k}\right)\right),
$$

where $P_{k}(\cdot)=P_{C_{k}}(\cdot)$.

Step 4. Choose real sequences $\left\{\alpha_{k}\right\},\left\{\beta_{k}\right\},\left\{\gamma_{k}\right\} \subset$
$(0,1)$ with $\alpha_{k}+\beta_{k}+\gamma_{k}=1(\forall k)$, and compute

$$
x^{k+1}=\alpha_{k} u+\gamma_{k} x^{k}+\beta_{k} t^{k} .
$$

\section{Remark 3.1.}

(1) When $\rho_{k}=1$, since $y^{k} \in K$, the iterative sequence $\left\{x^{k}\right\}$ generated by Algorithm 3.1 reduces to

$$
x^{k+1}=\alpha_{k} u+\gamma_{k} x^{k}+\beta_{k} J_{k}\left(x^{k}+e^{k}\right) .
$$

Furthermore, if $\gamma_{k}=0$, it reduces to

$$
x^{k+1}=\alpha_{k} u+\beta_{k} J_{k}\left(x^{k}+e^{k}\right) .
$$

(2) When $u=0 \in \mathcal{H}$ and $\rho_{k} \in[0,2)$, Algorithm 1.1 reduces to the generalized proximal point algorithm introduced by Zhang [6]; Furthermore, if $\rho_{k}=1$, then Algorithm 1.1 reduces to ones of Eckstein and Bertsekas [3].

(3) For any $k>0$, from the property (i) of $J_{k}$, it follows that $\left\|J_{k}\left(x^{k}+e^{k}\right)-J_{k}\left(x^{k}\right)\right\| \leq\left\|e^{k}\right\|$. Hence, (11) and (12) can be reformulated as

$$
x^{k+1}=\alpha_{k} u+\gamma_{k} x^{k}+\beta_{k} J_{k}\left(x^{k}\right)+\theta e^{k}
$$

and

$$
x^{k+1}=\alpha_{k} u+\beta_{k} J_{k}\left(x^{k}\right)+\theta e^{k}
$$

respectively, where $\theta \in(0,1)$. Therefore, when $\rho_{k}=1$, the iterative sequence $\left\{x^{k}\right\}$ generated by Algorithm 3.1 is equivalent to the iterative method suggested by Yao and Noor [15]. Furthermore, if $\gamma_{k}=0$, it reduces to the sequence given in Theorem 3 of Tian and Song [12].

Theorem 3.1. Let the set-valued mapping $M: \mathcal{H} \rightarrow 2^{\mathcal{H}}$ be maximal monotone, the sequences $\left\{x^{k}\right\},\left\{y^{k}\right\}$ and $\left\{t^{k}\right\}$ generated by Algorithm 3.1. If $\rho_{k} \in[0,1], \sum_{k=0}^{\infty}\left\|e_{k}\right\|<\infty$, then

(1) the sequences $\left\{x^{k}\right\},\left\{y^{k}\right\}$ and $\left\{t^{k}\right\}$ are bounded; Furthermore, $\lim _{k \rightarrow \infty}\left\|t^{k}-y^{k}\right\|=0$ when $\lim _{k \rightarrow \infty} \rho_{k}=1$;

(2) $\lim _{k \rightarrow \infty}\left\|y^{k}-x^{k}\right\|=0 \Leftrightarrow \lim _{k \rightarrow \infty}\left\|t^{k}-x^{k}\right\|=0$.

(3) when $\lim _{k \rightarrow \infty} \alpha_{k}=0$, we have

$$
\lim _{k \rightarrow \infty}\left\|t^{k}-x^{k}\right\|=0 \Rightarrow \lim _{k \rightarrow \infty}\left\|x^{k+1}-x^{k}\right\|=0 .
$$

(4) when $\lim _{k \rightarrow \infty} \alpha_{k}=0$ and $\liminf _{k \rightarrow \infty} \beta_{k}>0$, we have

$$
\lim _{k \rightarrow \infty}\left\|x^{k+1}-x^{k}\right\|=0 \Rightarrow \lim _{k \rightarrow \infty}\left\|t^{k}-x^{k}\right\|=0 .
$$

Proof. (1) Let $x^{*} \in \mathcal{H}$ be a solution of problem (1.1), then $0 \in M\left(x^{*}\right)$. By (9), we have

$$
\frac{1}{\lambda_{k}}\left(x^{k}-y^{k}+e^{k}\right) \in M\left(y^{k}\right) .
$$


By the monotonicity of $M$, we deduce that

$$
\left\langle 0-\frac{1}{\lambda_{k}}\left(x^{k}-y^{k}+e^{k}\right), x^{*}-y^{k}\right\rangle \geq 0,
$$

which leads to

$$
x^{*} \in C_{k}=\left\{z \in H:\left\langle x^{k}-y^{k}+e^{k}, z-y^{k}\right\rangle \leq 0\right\} .
$$

For $t^{k}=P_{k}\left(x^{k}-\rho_{k}\left(x^{k}-y^{k}\right)\right)$, by the property (P2) of the projection operator, we deduce that

$$
\begin{aligned}
\left\|x^{*}-t^{k}\right\| & \leq\left\|\left(1-\rho_{k}\right)\left(x^{k}-x^{*}\right)+\rho_{k}\left(y^{k}-x^{*}\right)\right\| \leq\left|1-\rho_{k}\right| \cdot\left\|x^{k}-x^{*}\right\|+\rho_{k}\left\|y^{k}-x^{*}\right\| \\
& \leq\left|1-\rho_{k}\right| \cdot\left\|x^{*}-x^{k}\right\|+\rho_{k}\left(\left\|x^{k}-x^{*}\right\|+\left\|e^{k}\right\|\right) \leq\left\|x^{*}-x^{k}\right\|+\left\|e^{k}\right\| .
\end{aligned}
$$

From (10) and (13), it follows that

$$
\begin{aligned}
\left\|x^{k+1}-x^{*}\right\| & =\left\|\alpha_{k}\left(u-x^{*}\right)+\gamma_{k}\left(x^{k}-x^{*}\right)+\beta_{k}\left(t^{k}-x^{*}\right)\right\| \\
& \leq \alpha_{k}\left\|u-x^{*}\right\|+\gamma_{k}\left\|x^{k}-x^{*}\right\|+\beta_{k}\left\|t^{k}-x^{*}\right\| \\
& \leq \alpha_{k}\left\|u-x^{*}\right\|+\left(\gamma_{k}+\beta_{k}\right)\left\|x^{k}-x^{*}\right\|+\beta_{k} \rho_{k}\left\|e^{k}\right\| \\
& \leq \alpha_{k}\left\|u-x^{*}\right\|+\left(1-\alpha_{k}\right)\left\|x^{k}-x^{*}\right\|+\left\|e^{k}\right\| .
\end{aligned}
$$

By induction we obtain

$$
\left\|x^{k+1}-x^{*}\right\| \leq \max \left\{\left\|u-x^{*}\right\|,\left\|x^{0}-x^{*}\right\|\right\}+\sum_{j=0}^{k}\left\|e^{j}\right\|, k>0 .
$$

Therefore, $\left\{x^{k}\right\}$ is bounded. Moreover, so are $\left\{y^{k}\right\}$ and $\left\{t^{k}\right\}$.

Furthermore, owing to $y^{k} \in C_{k}$, by the property (P2) of the projection operator, we have that

$$
\left\|t^{k}-y^{k}\right\| \leq\left|1-\rho_{k}\right| \cdot\left\|y^{k}-x^{k}\right\| .
$$

Therefore, when $\lim _{k \rightarrow \infty} \rho_{k}=1, \lim _{k \rightarrow \infty}\left\|t^{k}-y^{k}\right\|=0$.

(2) When $\rho_{k}=1$, it's obvious. Now for $\rho_{k} \neq 1$, since $t^{k}=P_{k}\left(x^{k}-\rho_{k}\left(x^{k}-y^{k}\right)\right)$ and $y^{k} \in C_{k}$, we have that

$$
\begin{aligned}
\left\|t^{k}-y^{k}\right\|^{2} & \leq\left\|\left(x^{k}-\rho_{k}\left(x^{k}-y^{k}\right)\right)-y^{k}\right\|^{2}-\left\|t^{k}-\left(x^{k}-\rho_{k}\left(x^{k}-y^{k}\right)\right)\right\|^{2}(\text { by }(P 3)) \\
& =\left(1-\rho_{k}\right)^{2}\left\|x^{k}-y^{k}\right\|^{2}-\left(1-\rho_{k}\right)^{2}\left\|t^{k}-x^{k}\right\|^{2}-\rho_{k}^{2}\left\|t^{k}-y^{k}\right\|^{2} \\
& -2 \rho_{k}\left(1-\rho_{k}\right)\left\langle t^{k}-x^{k}, t^{k}-y^{k}\right\rangle .
\end{aligned}
$$

Hence,

$$
\begin{aligned}
\left\|t^{k}-x^{k}\right\|^{2} & \leq\left\|y^{k}-x^{k}\right\|^{2}+\frac{2 \rho_{k}}{\left|1-\rho_{k}\right|}\left\langle t^{k}-x^{k}, y^{k}-t^{k}\right\rangle \\
& \leq\left\|y^{k}-x^{k}\right\|^{2}+2 \rho_{k}\left\|y^{k}-x^{k}\right\| \cdot\left\|t^{k}-x^{k}\right\|,
\end{aligned}
$$

and

$$
\left\|t^{k}-x^{k}\right\| \leq\left(\sqrt{1+\rho_{k}^{2}}+\rho_{k}\right)\left\|y^{k}-x^{k}\right\| \leq\left(2 \rho_{k}+1\right)\left\|y^{k}-x^{k}\right\| .
$$

This implies $\lim _{k \rightarrow \infty}\left\|y^{k}-x^{k}\right\|=0 \Rightarrow \lim _{k \rightarrow \infty}\left\|t^{k}-x^{k}\right\|=0$.

On the other hand, it follows from $t^{k} \in C_{k}$ that $\left\langle x^{k}+e^{k}-y^{k}, t^{k}-y^{k}\right\rangle \leq 0$ and

$$
\left\|x^{k}-y^{k}\right\|^{2} \leq\left\langle e^{k}, y^{k}-t^{k}\right\rangle+\left\langle y^{k}-x^{k}, t^{k}-x^{k}\right\rangle \leq\left\|e^{k}\right\| \cdot\left\|y^{k}-t^{k}\right\|+\left\|y^{k}-x^{k}\right\| \cdot\left\|t^{k}-x^{k}\right\| .
$$

Then, by (14), it holds that

$$
\left\|x^{k}-y^{k}\right\| \leq\left\|e^{k}\right\| \cdot\left|1-\rho_{k}\right|+\left\|t^{k}-x^{k}\right\| .
$$

Therefore, from the assumptions of $e_{k}$ and $\rho_{k}$, we have

$$
\lim _{k \rightarrow \infty}\left\|y^{k}-x^{k}\right\|=0 \Leftarrow \lim _{k \rightarrow \infty}\left\|t^{k}-x^{k}\right\|=0 .
$$


And so the equivalence relation holds.

(3) By the definition of $x^{k+1}$, we have

$$
\begin{aligned}
\left\|x^{k+1}-x^{k}\right\| & =\left\|\alpha_{k} u+\gamma_{k} x^{k}+\left(1-\alpha_{k}-\gamma_{k}\right) t^{k}-x^{k}\right\| \\
& =\left\|\alpha_{k}\left(u-t^{k}\right)+\left(1-\gamma_{k}\right)\left(t^{k}-x^{k}\right)\right\| \\
& \leq \alpha_{k}\left\|u-t^{k}\right\|+\left(1-\gamma_{k}\right)\left\|t^{k}-x^{k}\right\| .
\end{aligned}
$$

Hence, when $\lim _{k \rightarrow \infty} \alpha_{k}=0$ and $\lim _{k \rightarrow \infty}\left\|t^{k}-x^{k}\right\|=0$, we have

$$
\lim _{k \rightarrow \infty}\left\|x^{k+1}-x^{k}\right\|=0 .
$$

(4) Since $x^{k+1}-x^{k}=\beta_{k}\left(t^{k}-x^{k}\right)+\alpha_{k}\left(u-x^{k}\right)$, we have that

$$
\left\|t^{k}-x^{k}\right\| \leq \frac{1}{\beta_{k}}\left\|x^{k+1}-x^{k}\right\|+\frac{\alpha_{k}}{\beta_{k}}\left\|x^{k}-u\right\| .
$$

From $\lim _{k \rightarrow \infty} \alpha_{k}=0$ and $\liminf _{k \rightarrow \infty} \beta_{k}>0$, it follows that

$$
\lim _{k \rightarrow \infty}\left\|x^{k+1}-x^{k}\right\|=0 \Rightarrow \lim _{k \rightarrow \infty}\left\|t^{k}-x^{k}\right\|=0 .
$$

Lemma 3.1. If $\rho_{k} \in[0,1], \sum_{k=0}^{\infty}\left\|e_{k}\right\|<\infty$, and $\lim _{k \rightarrow \infty}\left\|t^{k}-x^{k}\right\|=0$, then $\Lambda\left(x^{k}\right) \subset S$, where $\Lambda\left(x^{k}\right)$ denotes the set of the weak cluster points of $\left\{x^{k}\right\}$ defined by Algorithm 3.1.

Proof. From Theorem 3.1(1), it follows that the sequence $\left\{x^{k}\right\}$ is bounded. So we can extract a subsequence that weakly converges to one weak accumulation point $\hat{x}$. Without loss of generality, let us suppose that $x^{k} \rightarrow \hat{x}$, then we have $y^{k} \rightarrow \hat{x}$. For any fixed $\nu \in \mathcal{H}$, take an arbitrary $\mu \in M(\nu)$. Then, it

Proof. By the definition of $x^{k}$ and the property of $\|\cdot\|$, we have that

$$
\left\|x^{k+1}-z\right\|^{2}=\left\|\alpha_{k}(u-z)+\beta_{k}\left(t^{k}-z\right)+\gamma_{k}\left(x^{k}-z\right)\right\|^{2} \leq\left\|\beta_{k}\left(t^{k}-z\right)+\gamma_{k}\left(x^{k}-z\right)\right\|^{2}+2 \alpha_{k}\left\langle u-z, x^{k+1}-z\right\rangle,
$$

and

$$
\left\|\beta_{k}\left(t^{k}-z\right)+\gamma_{k}\left(x^{k}-z\right)\right\|^{2}=\gamma_{k}\left(\gamma_{k}+\beta_{k}\right)\left\|x^{k}-z\right\|^{2}+\beta_{k}\left(\gamma_{k}+\beta_{k}\right)\left\|t^{k}-z\right\|^{2}-\gamma_{k} \beta_{k}\left\|x^{k}-t^{k}\right\|^{2} .
$$

Substituting (13)(replace $x^{*}$ by $z$ ) into (19), we have

$$
\left\|\beta_{k}\left(t^{k}-z\right)+\gamma_{k}\left(x^{k}-z\right)\right\|^{2} \leq\left(1-\alpha_{k}\right)\left\|x^{k}-z\right\|^{2}+\rho_{k} \beta_{k}\left(\gamma_{k}+\beta_{k}\right)\left(2\left\|x^{k}-z\right\|+\rho_{k}\left\|e^{k}\right\|\right)\left\|e^{k}\right\|-\gamma_{k} \beta_{k}\left\|x^{k}-t^{k}\right\|^{2} .
$$

Hence, we have that

$$
\begin{aligned}
\left\|x^{k+1}-z\right\|^{2} & \leq\left(1-\alpha_{k}\right)\left\|x^{k}-z\right\|^{2}+\rho_{k} \beta_{k}\left(\gamma_{k}+\beta_{k}\right)\left(2\left\|x^{k}-z\right\|+\rho_{k}\left\|e^{k}\right\|\right)\left\|e^{k}\right\| \\
& +2 \alpha_{k}\left\langle u-z, x^{k+1}-z\right\rangle-\gamma_{k} \beta_{k}\left\|x^{k}-t^{k}\right\|^{2}
\end{aligned}
$$

and

$$
\left\|x^{k+1}-z\right\|^{2} \leq\left(1-\alpha_{k}\right)\left\|x^{k}-z\right\|^{2}+M_{0}\left\|e^{k}\right\|+2 \alpha_{k}\left\langle u-z, x^{k+1}-z\right\rangle,
$$

where $M_{0}>0$ satisfying $\sup _{k>0}\left\{\beta_{k}\left(\gamma_{k}+\beta_{k}\right)\left(2 \rho_{k}\left\|x^{k}-z\right\|+\rho_{k}\left\|e^{k}\right\|\right)\right\} \leq M_{0}$. By the assumptions of $\left\{\lambda_{k}\right\}$ and $\left\{\beta_{k}\right\}$, there exists $\sigma>0$ such that $\sigma \leq \min \left\{\lambda_{k}, \gamma_{k} \beta_{k}\right\}$ for all $k>0$. So the following inequality holds:

$$
\left\|x^{k+1}-z\right\|^{2}-\left(1-\alpha_{k}\right)\left\|x^{k}-z\right\|^{2}+\sigma\left\|t^{k}-x^{k}\right\|^{2} \leq M_{0}\left\|e^{k}\right\|+2 \alpha_{k}\left\langle u-z, x^{k+1}-z\right\rangle .
$$

Setting $s_{k}=\left\|x^{k}-z\right\|^{2}$ for all $k \geq 0$, we arrive at

$$
s_{k+1}-\left(1-\alpha_{k}\right) s_{k}+\sigma\left\|t^{k}-x^{k}\right\|^{2} \leq M_{0}\left\|e^{k}\right\|+2 \alpha_{k}\left\langle u-z, x^{k+1}-z\right\rangle .
$$


In the sequel, we show $s_{k} \rightarrow 0$ by considering two cases on the sequence $\left\{s_{k}\right\}$.

Case $1\left\{s_{k}\right\}$ is eventually decreasing (i.e., there exists $N \geq$ 0 such that $\left\{s_{k}\right\}$ is decreasing for $\left.n \geq N\right)$.

In the case, $\left\{s_{k}\right\}$ must be convergent, and from (22) it follows that

$$
\begin{aligned}
\sigma\left\|t^{k}-x^{k}\right\|^{2} & \leq\left(1-\alpha_{k}\right) s_{k}-s_{k+1} \\
& +M_{0}\left\|e^{k}\right\|+2 \alpha_{k}\|u-z\| \cdot \sqrt{s_{k+1}} .
\end{aligned}
$$

By $\lim _{k \rightarrow \infty} \alpha_{k}=0$ and $\lim _{k \rightarrow \infty}\left\|e^{k}\right\|=0$, we have

$$
\lim _{k \rightarrow \infty}\left\|t^{k}-x^{k}\right\|=0 \text {. }
$$

So it follows from Lemma 3.1 that $\Lambda\left(x^{k}\right) \subset S$. Choose a subsequence $\left\{x^{k_{j}}\right\}$ of $\left\{x^{k}\right\}$ such that

$$
\limsup _{k \rightarrow \infty}\left\langle u-z, x^{k}-z\right\rangle=\limsup _{j \rightarrow \infty}\left\langle u-z, x^{k_{j}}-z\right\rangle,
$$

and $x^{k_{j}} \rightarrow \tilde{x}$.

Since $\tilde{x} \in S$, we get that

$$
\limsup _{k \rightarrow \infty}\left\langle u-z, x^{k}-z\right\rangle=\left\langle u-P_{S}(u), \tilde{x}-P_{S}(u)\right\rangle \leq 0 .
$$

Applying Lemma 2.1 to (21), we conclude that $\left\{x^{k}\right\}$ converges strongly to the point $z=P_{S}(u)$.

Case $2\left\{s_{k}\right\}$ is not eventually decreasing. Hence, there exists a subsequence $\left\{s_{k_{j}}\right\}$ such that $s_{k_{j}} \leq s_{k_{j}+1}$ for all $j \geq 0$. So, an integer sequence $\left\{\tau_{k}\right\}$ can be defined as in Lemma 2.2. From Lemma 2.2, it follows that $s_{\tau_{k}} \leq s_{\tau_{k}+1}$ for all $k>k_{0}$. Therefore, by (22), we have that

$$
\begin{aligned}
\sigma\left\|t^{\tau_{k}}-x^{\tau_{k}}\right\|^{2} & \leq M_{0}\left\|e^{\tau_{k}}\right\| \\
& +2 \alpha_{\tau_{k}}\|u-z\| \cdot \sqrt{s_{\tau_{k}+1}}, \quad \forall k>k_{0} .
\end{aligned}
$$

By $\lim _{k \rightarrow \infty} \alpha_{k}=0$ and $\lim _{k \rightarrow \infty}\left\|e^{k}\right\|=0$, we have

$$
\lim _{k \rightarrow \infty}\left\|t^{\tau_{k}}-x^{\tau_{k}}\right\|=0
$$

In a similar way as in case 1 we deduce that $\Lambda\left(x^{\tau_{k}}\right) \subset S$, and

$$
\limsup _{k \rightarrow \infty}\left\langle u-z, x^{\tau_{k}}-z\right\rangle \leq 0 .
$$

So, by Theorem 3.1(3), we have

$$
\limsup _{k \rightarrow \infty}\left\langle u-z, x^{\tau_{k}+1}-z\right\rangle \leq 0 .
$$

By (21), we also have

$s_{\tau_{k}+1} \leq\left(1-\alpha_{\tau_{k}}\right) s_{\tau_{k}}+M_{0}\left\|e^{k}\right\|+2 \alpha_{k}\left\langle u-z, x^{\tau_{k}+1}-z\right\rangle$.

Hence, it follows from Lemma 2.1 that $\lim _{k \rightarrow \infty} s_{\tau_{k}+1}=0$. Since $\max \left(s_{\tau_{k}}, s_{k}\right) \leq s_{\tau_{k}+1}$ for all $k>k_{0}$, we conclude that $\lim _{k \rightarrow \infty} s_{k}=0$, i.e., $\lim _{k \rightarrow \infty} x^{k}=z$. This completes the proof. $\diamond$

Theorem 3.3. Let the set-valued mapping $M: \mathcal{H} \rightarrow 2^{\mathcal{H}}$ be maximal monotone, $u \in \mathcal{H}$ be a given point. Select initial $x^{0} \in \mathcal{H}$, the iterative sequence $\left\{x^{k}\right\}$ generated by

$$
x^{k+1}=\alpha_{k} u+\gamma_{k} x^{k}+\beta_{k} J_{k}\left(x^{k}+e^{k}\right),
$$

where $\alpha_{k}, \beta_{k}, \gamma_{k} \subset(0,1)$ with $\alpha_{k}+\beta_{k}+\gamma_{k}=1(\forall k)$ are real sequences, the number $\lambda_{k}>0$ and $e^{k}$ is an error. If

(1) $\sum_{k=0}^{\infty} \alpha_{k}=\infty, \lim _{n \rightarrow \infty} \alpha_{k}=0, \liminf _{k \geq 0} \lambda_{k}>0$,

(2) $1>\lim \sup _{k \geq 0} \beta_{k} \geq \liminf \operatorname{in}_{k \geq 0} \beta_{k}>0$, $\sum_{k=0}^{\infty}\left\|e_{k}\right\|<\infty$.

Then the sequence $\left\{x^{k}\right\}$ is convergent strongly to $z=$ $P_{S}(u)$.

Proof. Let $\rho_{k}=1$ in Algorithm 3.1, then we have $t^{k}=y^{k}$ for all $k \geq 0$. It follows from $y^{k}=J_{k}\left(x^{k}+e^{k}\right) \in K$ and Theorem 3.2 that the conclusion holds. $\diamond$

\section{Numerical Example}

In this section, we give some numerical examples to show our results.

Let $\mathcal{H}=R, M: R \rightarrow 2^{R}$ defined by $M(x)=\{x\}$. It is obvious that $M$ is maximal monotone and $S=\{0\} \neq \emptyset$.

Take

$$
u=\frac{1}{2}, x^{0}=\frac{1}{2}, e^{k}=0(\forall k \geq 0), \lambda_{k}=1+\frac{1}{k+1}(\forall k \geq 0),
$$

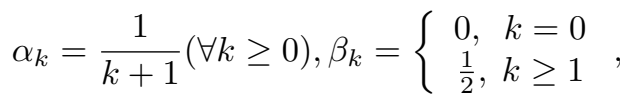

then

$$
\gamma_{k}=\left\{\begin{array}{c}
0, k=0 \\
\frac{k-1}{2(k+1)}, k \geq 1
\end{array}\right.
$$

There are some iterative points generated by Algorithm 3.1 which is shown in Table 1 for the example above. From Table 1, it's obvious that the convergent speed of Algorithm 3.1 for $\rho_{k}=1$ is slower than the other case, the convergent speed of Algorithm 3.1 for $\rho_{k}=\frac{1}{2}$ is faster. If $x^{k}=0.01294$, it gets at 102th iterative steps for $\rho_{k}=\frac{1}{2}$, but it gets at about 154th iterative steps for $\rho_{k}=1$.

Table 1. Some iterative points generated by Algorithm 3.1.

\begin{tabular}{cccccccccc}
\hline $\mathrm{k}$ & 50 & 100 & 200 & 500 & 800 & 1000 & 1100 & 1200 \\
\hline$\rho=1$ & 0.03956 & 0.01990 & 0.00997 & 0.00400 & 0.00250 & 0.00200 & 0.00182 & 0.00167 \\
$\rho=\frac{1}{2}$ & 0.02603 & 0.01318 & 0.00663 & 0.00266 & 0.00166 & 0.00133 & 0.00121 & 0.00111 \\
\hline
\end{tabular}


The following two figures(Figure 1, Figure 2) show the convergence of the sequence $\left\{x^{k}\right\}$ given in the example above visually. From the figures, we can know the convergence speed is not affected by the projection in Algorithm 3.1 in this example.

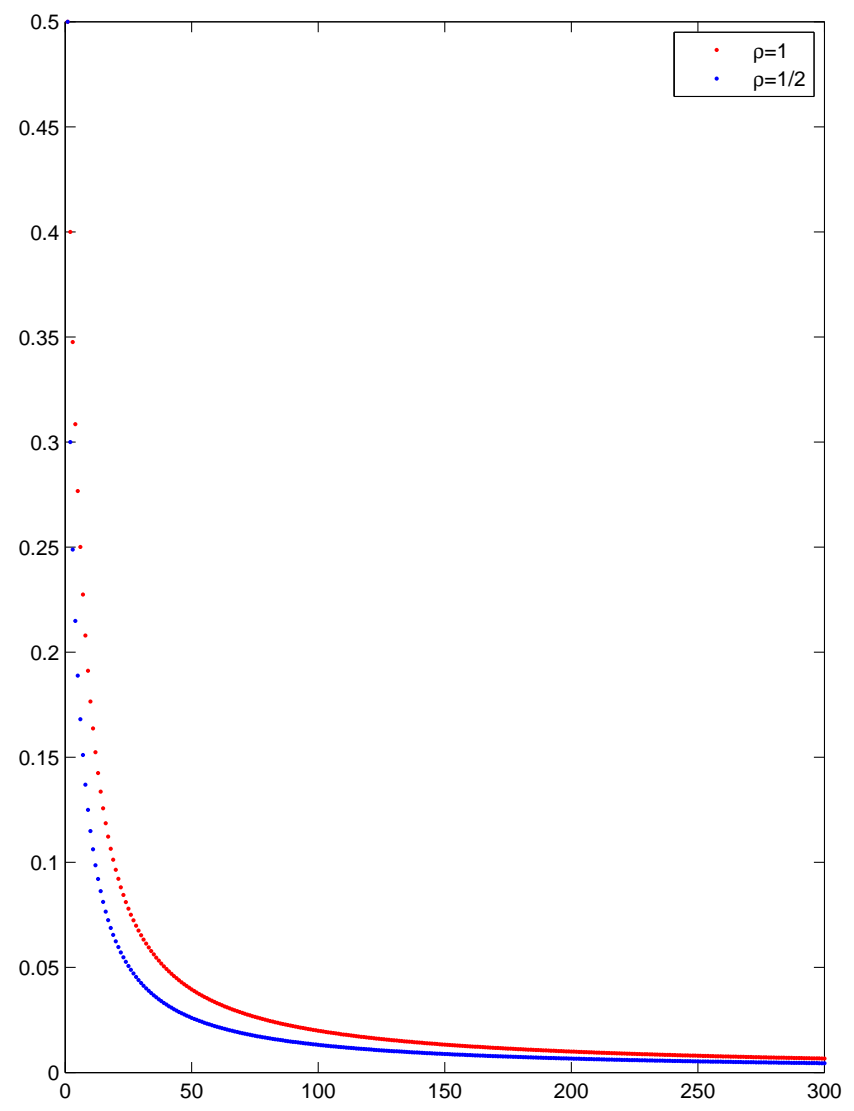

Figure 1. Iterative points generated by Algorithm 1.1 from 1 to 300 .

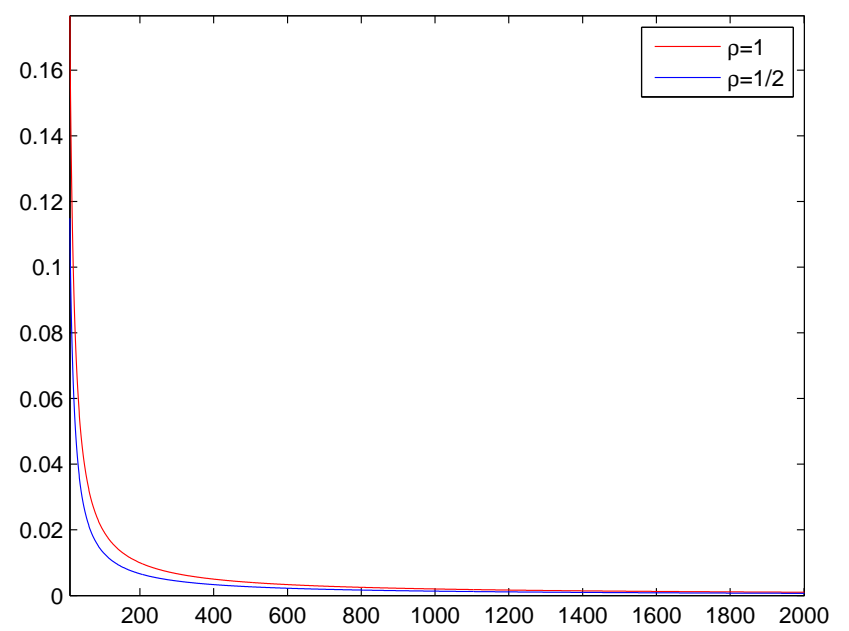

Figure 2. Iterative points generated by Algorithm 1.1 from 1 to 2000.

Remark 4.1. When $e^{k}=0(\forall k \geq 0)$, Theorem 3.3 of Yao and Noor ([15]) is same as Theorem 3.3. But the convergence speed of Algorithm 3.1 for $\rho_{k} \neq 1$ is quicker than that given in Theorem 3.3 of Yao and Noor ([15]) in this numerical example. Hence, the convergence and the convergence speed of Algorithm 3.1 is improved by the projection.

\section{Conclusions}

In this paper, we construct a hybrid Halpern type proximal point algorithm with errors, which combinate the Halpern type proximal point algorithm [15] and the modified proximal point algorithm with errors [6], for approximating the zero of a maximal monotone operator, and prove the strong convergence of the given algorithm with weaker assumptions in Hilbert space. Hence, we give an affirmant answer of the strong convergence of the modified proximal point algorithm with errors by using a modified orthogonal projection onto a moving halt-spaces of Zhang [6], and generalize the corresponding results of Yao and Noor [15] and the others. In addition, future research will consider the generalization of our results in a uniformly convex Banach space with a uniformly Gâteaux differentiable norm for approximating the zero of a m-accretive operator, which would generalize the corresponding results of Zhang and Song [17]. On the other hand, because Leuştean, Nicolae and Sipoş[22] discussed the application of the proximal point algorithm in $C A T(0)$ space, and Gheorghe and Adrian[23] extended the proximal point algorithm in Hilbert space, we would generalized and improved our algorithm in $C A T(0)$ space.

\section{Acknowledgements}

This work is supported partially by the Scientific Research Fund of the Southwestern University of Finance and Economics. The authors are grateful to the referees for the careful reading and the valuable suggestion.

\section{References}

[1] R. T. Rockafellar, Monotone operators and the proximal point algorithm, SIAM J. Control Optim., 14 (1976), 877 898.

[2] O. Güler, On the convergence of the proximal point algorithm for convex optimization, SIMA J. Control. Optim., 29 (1991), 403-419.

[3] J. Eckstein, D. P. Bertsekas, On the Douglas-Rachford splitting method and the proximal point algorithm for maximal monotone operators, Math. Programming, 55 (1992), 293-318.

[4] B. S. He, L. Liao, Zh. Yang(2003). A new approximate proximal point algorithm for maximal monotone operator, Sci. China Ser. A, 46 (2): 200-206. 
[5] Zh. Yang, B. S. He, A Relaxed Approximate Proximal Point Algorithm, Ann. Oper. Res., 133 (2005), 119-125.

[6] Q.B. Zhang, A modified proximal point algorithm with errors for approximating solution of the general variational inclusion, Operat. Research Lett., 40 (2012), 564-567.

[7] Y. Liu, Weak convergence of a hybrid type method with errors for a maximal monotone mapping in Banach spaces, J. Inequal. Appl., 2015 (2015), 260.

[8] O. A. Boikanyo, G. Morosanu, Four parameter proximal point algorithms, Nonlinear Anal., 74 (2011), 544-555.

[9] H. Khatibzadeh, S. Ranjbar, On the Strong Convergence of Halpern Type Proximal Point Algorithm, J. Optim. Theory Appl., 158 (2) (2013), 385-396.

[10] T. H. Kim, H. K. Xu, Strong convergence of modified mann iterations, Nonlinear. Anal., 61 (2005), 51-60.

[11] S. Saejung, A supplement to a regularization method for the proximal point algorithm, J. Glob. Optim., 56 (2013), 121-129.

[12] Ch. Tian, Y. Song, Strong convergence of a regularization method for Rockafellar's proximal point algorithm, J. Glob. Optim., 55 (2013), 831-837.

[13] F. Wang, H. Cui, On the contraction proximal point algorithms with multi parameters. J. Glob. Optim., 54 (3) (2012), 1-7.

[14] H. K. Xu, Strong convergence of an iterative method for non expansive and accretive operators, J. Math. Anal. Appl., 314 (2006), 631-643.
[15] Y. Yao, M. A. Noor, On convergence criteria of generalized proximal point algorithms, J. Comput. Appl. Math., 217 (2008), 46-55.

[16] H. K. Xu, A regularization method for the proximal point operators, J. Glob. Optim., 36 (2006), 115-125.

[17] Q. N. Zhang, Y. S. Song, Halpern type proximal point algorithm of accretive operators, Nonlinear Appl., 75 (4) (2012), 1859-1868.

[18] G. Marino, H. K. Xu, Convergence of generalized proximal point algorithm, Comm.Pure Appl. Anal., 3 (2004), 791-808.

[19] M. A. Moor, Projection-proximate methods for general variational inequalities, J. Math. Anal. Appl., 318 (2006), 53-62.

[20] H. K. Xu, Iterative algorithms for nonlinear operators, J. Londen Math. Soc., 66 (2002), 240-256.

[21] P. E. Maingé, Strong convergence of projected subgradient methods for nonsmooth and nonstrictly convex minimization, Set-Valued Anal., 16 (2008), 899912.

[22] L. Laurenţiu, N. Adriana, S. Andrei, An abstract proximal point algorithm, J. Global Optim., 72 (3) (2018), 553-577.

[23] M. Gheorghe, P. Adrian, A proximal point algorithm revisited and extended, J. Optim. Theory Appl., 182 (3) (2019), 1120-1129. 